\title{
A review of codebook design methods for sparse code multiple access
}

\author{
Syed Aamer Hussain ${ }^{1}$, Norulhusna Ahmad ${ }^{2}$, Ibraheem Shayea ${ }^{3}$, Hazilah Mad Kaidi ${ }^{4}$, Liza Abdul \\ Latiff $^{5}$, Norliza Mohamed ${ }^{6}$, Suriani Mohd Sam ${ }^{7}$ \\ 1,2,4,5,6,7 Razak Faculty of Technology and Informatics, Universiti Teknologi Malaysia (UTM), Malaysia \\ ${ }^{3}$ Faculty of Electrical and Electronics Engineering, Istanbul Technical University (ITU), Turkey
}

\begin{tabular}{l} 
Article Info \\
\hline Article history: \\
Received Dec 6, 2020 \\
Revised Mar 23, 2021 \\
Accepted Mar 29, 2021 \\
\hline
\end{tabular}

Keywords:

5G mobile communication

NOMA

SCMA

\begin{abstract}
The progressions in telecommunication beyond the 5th generation have created a need to improve research drifts. The current $5 \mathrm{G}$ study has an important focus on non-orthogonal multiple access (NOMA) technology. Sparse code multiple access (SCMA) is a promising technique within NOMA, enhancing the multi-user handling capability of next-generation communication. In the SCMA sphere, codebook designing and optimisation are essential research matters. This study conversed with different codebook design practises existing in the literature, analysing them for numerous parameters, including bit error rate (BER), an optimisation technique, and channel settings. From the analysis, the paper presents the efficiency of different approaches. The article also discusses the prospects and challenges of SCMA optimisation in practical implementation in various domains.
\end{abstract}

This is an open access article under the CC BY-SA license.

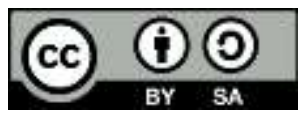

\section{Corresponding Author:}

Norulhusna Ahmad

Razak Faculty of Technology and Informatics

UTM, Kuala Lumpur, Malaysia

Email: norulhusna.kl@utm.my

\section{INTRODUCTION}

Wireless communication has gone through four significant generations, incorporating advancements in different parameters and improving the system's capability [1]-[2]. Each generation has included new ways for spectral efficiency with the best possible benchmarks [3]. Different multiplexing techniques are proposed in each generation (1G to $4 \mathrm{G}$ ) [4], evolving to the current orthogonal frequency division multiple access (OFDMA) [5]-[6]. The orthogonal multiple access (OMA) idea exists in dividing single narrowband frequency resource among different users orthogonally. The technique allows the mitigation of interference among various users and improving communication reliability. However, the division of time/frequency resources among users has limited spectrum utilisation and connectivity capability. Non-orthogonal multiple access (NOMA) techniques exist in modern telecommunication systems [7]-[8]. NOMA assigns resources and users on a sharing basis rather than division. The sharing allows improved utilisation of a single resource, thereby improving spectral efficiency [9].

Like OMA, NOMA has its own set of techniques, categorised based on the type of sharing mechanism used. Mainly classified into code-domain, power domain, and multiple domain NOMA [10]. The power domain NOMA is a single carrier technique multiplexing various users in the same resource block (RB) by varying their power levels based on individual channel conditions [11] separated at the receiver through successive interference cancellation (SIC) technique [12]. Code domain NOMA includes multi-carrier techniques and can accommodate multiple users within the same RB based on codes. The main code domain techniques are low- 
density spreading (LDS) and sparse code multiple access (SCMA). The difference among the two exists in the data encoding, where SCMA uses multidimensional (MD) constellations to map the input bits into codewords. LDS uses repetition of QAM symbols over all the assigned RBs while SCMA uses different symbols for each $\mathrm{RB}$, making it more robust and complex. Multiple-domain NOMA incorporates power, code, and spatial domain in multiplexing user data [13]. Prominent techniques are pattern division multiple access (PDMA) and lattice partition multiple access (LPMA). PDMA sparsely spread user data on the assigned RBs like SCMA [14]. LPMA uses code and power domains combined for user data multiplexing [15]. A comparison between the power and code domain SCMA concludes that code domain SCMA has a much higher complexity in receiver architecture [14]. Research also shows that within the multi-domain NOMA and SCMA, SCMA has a much better performance than PDMA because of the MD constellation design [16]. SCMA is found to have higher robustness, massive connectivity [17], low latency [18] and is also a better option for uplink communication [19].

This paper reviews the design considerations in SCMA architecture, focusing on the codebook designing for different modulation schemes and their effect on performance. The paper also presents the BER analysis of different codebooks. A similar comparative analysis is conducted by Vameghestahbanati [20]; however, that study's primary focus is channel coding and path loss models. The article has six sections. Section 1 introduces the SCMA system. Section 2 discusses the SCMA architecture, and Section 3 describes the codebook design methods. Section 4 discuss the performance of each technique. The last section presents the future challenges and conclusion of the study.

\section{SYSTEM ARCHITECTURE}

SCMA is a non-orthogonal technique primarily based on the MD codebook. The architecture of SCMA consists of encoder and decoder stages using codebook mapping. The basic block diagram of the SCMA system is shown in Figure 1 [12].

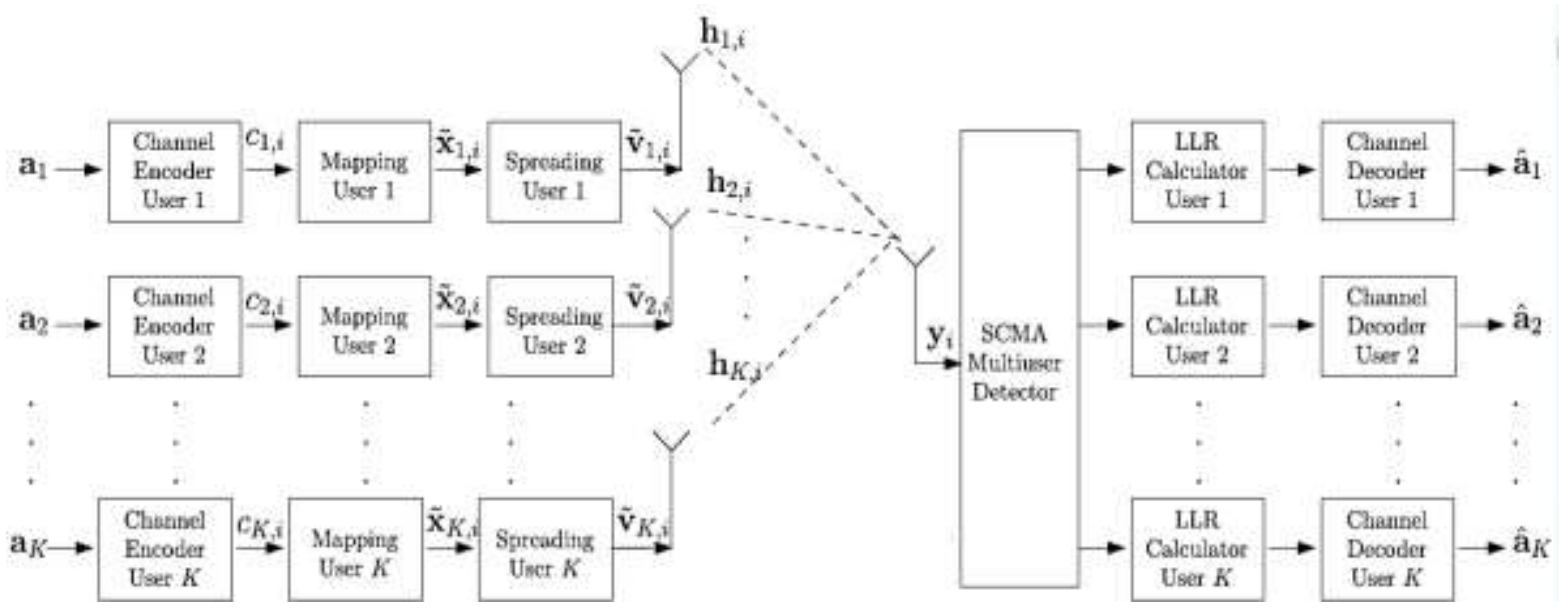

Figure 1. SCMA architecture

Consider an SCMA system with $K$ number of users multiplexed at $N$ orthogonal resources, where $N<K$. Each user's data is sent through $d_{v}$ number of RBs where $d_{v}<<N$. In the SCMA system, initially, the user data bitstream is passed to the error correction encoder, which reduces noise and interference effect on the transmitted data and minimises the bit errors [22]-[24]. The encrypted data enters the SCMA encoder block consisting of mapper and spreading sub-blocks. Mapping allows the spreading of user data in the complex domain by adding phase and amplitude of constellation points to different data values from the same or different users. SCMA mapper maps each symbol to the $d_{v}$ dimensional constellation point producing codeword of length $N_{c}$. In $M$ point signal constellation of each user each $L_{M}=$ $\log _{2} M$ bits are represented by one constellation point. Also, Codewords from the channel encoder are divided into $N_{c u}=N_{c} / L_{M}$ where, $N_{c u}$ represent the number of channels required to transmit one codeword. Encoder outputs $c_{(k, i)}$ symbol as binary numbers $\left(k \in(1,2, \ldots, K)\right.$ and $i \in\left(1,2, \ldots, N_{c u}\right)$ where $i$ is the digital symbol of user $k$. SCMA mapper maps the incoming data to $d_{v}$ dimensional constellation points to 
produce. $x_{k, i}=\left(x_{1, k, i}, x_{2, k, i}, \ldots, x_{d_{v}, k, i}\right)$. For mapping, the constellation point is selected from a complex constellation of $X_{k}=\left\{x_{k, m}\right\}$ where $m \in(1,2, \ldots, M)$. Apart from mapping, the SCMA also spreads the data for sparsity. Each constellation point $\mathbf{x}_{k, I}$ is coded into a sparse of $N$-dimensional complex Codeword $\mathbf{v}_{\mathrm{k}, \mathrm{I}}$ through user to RE spreading matrix $\mathrm{S}_{\mathrm{k}} \cdot \mathbf{v}_{k, I}$ are sparse vector and a member of the sparse codebook $\mathrm{V}_{\mathrm{k}}$ is given by (1).

$$
V_{k}=v_{k, m}=x_{k, m} S_{k}
$$

For handling spreading, a matrix is created governing how multiple user data needs to be multiplexed on each RB. In case of regular spreading for a single user out of $N$ occupying $d_{v}=2$ resources binary user to $\mathrm{RB}$ spreading matrix is given by (2). The complete binary user to RE matrix is an $N \times K d_{v}$ matrix denoted as $S=\left[S_{1}, S_{2}, \ldots, S_{K}\right]$. Therefore, for $K=6, N=4$ and $d_{v}=2$ the $4 \times 12$ users of the RB matrix are given by (3).

$$
\begin{aligned}
& \mathrm{S}_{\mathrm{i}}=\left[\begin{array}{ll}
0 & 0 \\
1 & 0 \\
0 & 0 \\
0 & 1
\end{array}\right] \text { where }(\mathrm{i}=1,2, \ldots, \mathrm{N}) \\
& {\left[\begin{array}{llllllllllll}
0 & 0 & 1 & 0 & 1 & 0 & 0 & 0 & 1 & 0 & 0 & 0 \\
1 & 0 & 0 & 0 & 0 & 1 & 0 & 0 & 0 & 0 & 1 & 0 \\
0 & 0 & 0 & 1 & 0 & 0 & 1 & 0 & 0 & 0 & 0 & 1 \\
0 & 1 & 0 & 0 & 0 & 0 & 0 & 1 & 0 & 1 & 0 & 0
\end{array}\right]}
\end{aligned}
$$

The resource sharing among the users is governed by sparse matrixes called mapping matrix. The sparse nature of these matrixes protects data from corruption and facilitates decoding. Allocation of $d_{v}$ REs to $K$ users is done through $N$-dimensional user to RE indicator vector given by $f_{k}=\operatorname{diag}\left(S_{k} S_{k}^{T}\right)$ where $f_{k}=$ 1 means RE is assigned to the user. Combining the vectors form an indicator matrix $\mathrm{F}=\left[f_{1} \ldots f_{k}\right]$ as given in (4).

$$
F_{-}=\left[\begin{array}{llllll}
1 & 1 & 1 & 0 & 0 & 0 \\
1 & 0 & 0 & 1 & 1 & 0 \\
0 & 1 & 0 & 1 & 0 & 1 \\
0 & 0 & 1 & 0 & 1 & 1
\end{array}\right]
$$

In the case of uplink, the codeword $v_{k, i}$ for each user is transmitted on a separate uplink channel having different channel coefficients, while for downlink, the channel coefficient is the same for all channels. The received signal $y_{i}$ considered a Rayleigh fading channel in uplink is given by (5) [25].

$$
\mathrm{y}_{\mathrm{i}}=\sum_{\mathrm{k}=1}^{\mathrm{K}} \operatorname{diag}\left(\mathrm{h}_{\mathrm{k}, \mathrm{i}}\right) \mathrm{v}_{\mathrm{k}, \mathrm{i}}+\mathrm{w}_{\mathrm{i}}
$$

Where the channel coefficient for the Rayleigh fading channel in uplink is $h_{k, i}=\left(h_{1, k, i}, h_{2, k, i}, \ldots, h_{N, k, i}\right)^{T}$ and $\mathrm{w}_{i}$ is a zero-mean complex Gaussian noise with a variance of one and [. $]^{T}$ is the transpose operation [26].

In SCMA, various algorithms are deployed for decoding, including MPA, log MPA, peeling decoder to evaluate the received data [27]. MPA is a complex algorithm with many exponential operations involved [28] Max-log-MPA [26] and log-MPA [27] reduce the complexity of the process by using logarithmic domain operations in calculations. A relatively uncomplicated approach with adequate performance is with the peeling decoder, which operates by sequentially extracting user data [29].

\section{SCMA MULTIDIMENSIONAL CONSTELLATION AND CODEBOOK DESIGN}

The main performance parameter to analyse any communication system is its reliability through BER. The BER of SCMA systems is mainly based on the codebook. The codebook design procedure involves two phases: the MD constellation design and arithmetic operations on MD constellation for codebook generation. The goal of designing mother constellation (MC) and optimisation is to maximise the minimum euclidean distance (MED) and to have energy diversity (ED). Both are dependent variables; increasing energy diversity in the MD constellation reduces the MED and vice versa, so a conciliation is made in designing. 


\subsection{Multidimensional constellation design}

A modulation scheme needs to be designed for encoding the user data. The requirement is to have maximum Euclidean distance and energy diversity between the points. Generally, the SCMA structure can be represented as a function of $\tau\left(S, X, K, M, d_{v}, N\right)$ where $X$ is the MD constellation, not considering the constants, the SCMA optimisation equation is based on spreading matrix $S$ and constellation $X$ given by (6).

$$
S, X=\arg \max _{S, X} D(\tau)
$$

For a fully loaded system, the spreading matrix has a unique solution, and the optimisation equation from (6) is reduced to a constellation operator. Based on (6), constellation design is primarily performed in two steps, first design a mother constellation of size $\mathrm{M}$ and then perform user-specific rotation operations to generate user-specific MD constellations. These operations maintain the uniqueness of symbols for users that are transmitted in the same RE. However, in the uplink, the channel applies a random rotation to constellations, so the rotation does not affect the system's output. But, it allows the critical performance indicators to be configured for better uplink SCMA performance.

\subsection{Codebook designing}

The codebook design performs layer-specific operations $\phi$ on the MD constellation matrix, $M C B=$ $M C * \phi$, where $M C$ is the optimised MD constellation. Special operations involve in the codebook designing are complex conjugate, phase rotation and vector permutation. The designing can be a combination of these operations depending upon the performance enhancement of SCMA in BER improvement. User codebooks are extracted from the mother codebook using the mapping matrixes of the factor graph. Element wise product of codebook and each user vector-matrix generates the user codebooks. For $K$ number of users, each with the codebook $C B_{K}$ the mother codebook matrix is defined as $C B=\left[\begin{array}{lllll}C B_{1} & C B_{2} & \cdot & \cdot & C B_{K}\end{array}\right]^{T}$.

In codebook design, the constellation point separation is necessary for Additive white Gaussian noise (AWGN) transmission. There is a need for energy diversity and product distance between the constellation points for performance enhancement in a fading channel. The improvement is made using the phase rotation technique. The phase rotation is carried out in two steps; firstly, the constellation used in communication is partitioned into sub-constellations of $1,2,4$ or 8 points. Next, the rotation parameter $\theta$ needs to be calculated by maximising the $\theta$ as in (7), for which optimisation is performed on the minimum coordinate product distance (CPD) for all the sub-constellations created in step 1 given by (8).

$$
\begin{aligned}
& \Theta=\max _{\Theta}\left(\min \left(\mathrm{CPD}_{1}, \mathrm{CPD}_{2}, \ldots, \mathrm{CPD}_{\mathrm{m}_{\mathrm{s}}}, \ldots, \mathrm{CPD}_{\mathrm{M}_{\mathrm{s}}}\right)\right), \\
& \mathrm{CPD}=\prod_{\mathrm{k}=1}^{\mathrm{D}_{\mathrm{c}}}\left(\mathrm{c}_{\mathrm{k}}^{\mathrm{a}}-\mathrm{c}_{\mathrm{k}}^{\mathrm{b}}\right)
\end{aligned}
$$

Where $1 \leq m_{s} \leq M_{s}$ in which $M_{s}$ is the total number of constellations created by division and $D_{C}$ are the coordinates between two constellation points $a$ and $b$ with dimensions $c_{k}^{a}$ and $c_{k}^{b}(k$ represent the number of dimensions). The other layer operation that can be performed is the coordinate interleaving [30]. It uses both $I$, and $Q$ coordinates interleave. The process of interleaving is to reorder the elements of even dimensions (rows). Permutation operations are also performed on the codebook to capture the maximum sum of the distance between dimensions of codewords sharing an RB. The operation results in a diversity of energy among the interfering codewords' dimensions multiplexed on all the RBs.

\section{CODEBOOK DESIGN METHODS}

There are many techniques to optimise the constellations for codebook design. These methods can be classified into three categories lattice-based optimisation, constellation rotation with permutation, and factor graph optimisation. AWGN and rayleigh fading channels both exist in the simulation of these techniques. Table 1 reviews the effectiveness of the methods in different environments based on the results claimed.

a) Lattice-based optimisation: In the category of lattice-based optimisation, Zhang presented lattice optimisation theory-based constellation for codebook design achieving better coding gain [31] with an overloading coefficient of $250 \%$. The study compared the method with other methods, but the comparison does not include channel modelling and fading, so the results will be considered ideal. Dong [32] presented a superposition method incorporating repetitive optimisation technique with the line search algorithm for codebook design. The method using turbo channel coding is found better in mid and low range signal to noise ratios (SNRs); however, significant comparative results at higher SNR does not 
exist. Since the study uses a small block size, polar codes can also be used for faster operation without compromising the error rate. Zhang [33] researched another lattice optimisation mechanism where MD constellations are designed by combining the optimised 1-dimensional constellations through rotation angles, thereby improving the sum rate. The method incorporated LDPC coding in AWGN and fading channel. Since the research considers a high sum-rate in its operation, LDPC coding is recommended; otherwise, the technique can also be tested with turbo coding for moderate rates. Another approach towards codebook designing using lattice improvements is based on a constant modulus approach improving mutual information [33]. It claims to have a 39\% inter-resource block distance improvement. Considering the AWGN channel, the method deploys a decent gradient algorithm for getting optimised parameters. However, the decent gradient algorithm is relatively crude in complex constellation scenarios where multiple local minima and outlier can alter the results. The study presented no such mechanisms to avoid such cases; however, it is suggested to include adaptive or other stochastic approaches in optimising the results. Like [34], Bao proposed designing MD constellations from single-dimensional but low projected points [35]. Here MD permutations are performed for generating higher dimensional constellations. The method incorporated interleaving in its operation and is tested under AWGN and fading channels. Since multistage optimisation is introduced for constellation design, BER performance is considerably improved.

Table 1. Codebook design methods

\begin{tabular}{|c|c|c|c|c|}
\hline Method & Ref & Method Improvements & Performance & Channel Info \\
\hline \multirow[t]{5}{*}{$\begin{array}{c}\text { Lattice- } \\
\text { Based } \\
\text { Optimisation }\end{array}$} & [31] & $\begin{array}{l}\text { Lattice-Based Codebook Design: Uses lattice optimisation } \\
\text { theory for achieving MED, providing coding gain with } \\
\text { power diversity in interfering codewords for easy user } \\
\text { separation. }\end{array}$ & $\begin{array}{l}\text { Achieves } 10^{-4} \text { BER } \\
\text { with }>28 \mathrm{~dB} \text { SNR }\end{array}$ & $\begin{array}{l}\text { AWGN } \\
\text { channel with } \\
\text { no coding }\end{array}$ \\
\hline & [32] & $\begin{array}{l}\text { Codebook Optimisation Based on Mutual Information } \\
\text { Maximization: Superposition model with line search for } \\
\text { optimal codebook searching with better results in AWGN } \\
\text { low/medium SNR }\end{array}$ & $\begin{array}{l}\text { Achieves } 10^{-4} \text { BER } \\
\text { with } 17.5 \mathrm{~dB} \text { SNR }\end{array}$ & $\begin{array}{l}\text { AWGN } \\
\text { channel with } \\
\text { turbo coding }\end{array}$ \\
\hline & [34] & $\begin{array}{l}\text { Capacity-based codebook design method: Construction of } \\
\text { MED optimised codebook using Latin square criterion }\end{array}$ & $\begin{array}{lll}\text { Achieves } & 10^{-4} & \text { BER } \\
\text { 5dB SNR } & & \end{array}$ & $\begin{array}{l}\text { AWGN and } \\
\text { fading channel }\end{array}$ \\
\hline & [33] & $\begin{array}{l}\text { Constant Modulus Codebook Design: Uses Gradient } \\
\text { descent with constant modulus criterion for MED } \\
\text { optimised codebook }\end{array}$ & $\begin{array}{l}\text { It has a } 39 \% \text { MED } \\
\text { improvement }\end{array}$ & $\begin{array}{l}\text { AWGN } \\
\text { Channel }\end{array}$ \\
\hline & [35] & $\begin{array}{l}\text { Bit-interleaved coded SCMA: Achieved improvement in } \\
\text { MED by low projected MD constellation for fading } \\
\text { channel using a multistage optimisation scheme }\end{array}$ & $\begin{array}{l}\text { Achieves } 10^{-4} \text { BER for } \\
E_{b} / N_{o}=5 \text { for } 4 \text { array, } \\
6.5 \text { for } 8 \text { array, and } 6 \\
\text { for } 16 \text { array codebooks }\end{array}$ & $\begin{array}{l}\text { AWGN and } \\
\text { Fading channel } \\
\text { (convolutional } \\
\text { coding) }\end{array}$ \\
\hline $\begin{array}{l}\text { Constellation } \\
\text { Rotation } \\
\text { with }\end{array}$ & [7] & $\begin{array}{l}\text { Codebook design based on constellation rotation and } \\
\text { interleaving: Improves MED by constellation point's } \\
\text { rotation and codewords interleaving }\end{array}$ & $\begin{array}{l}\text { Achieves } 10^{-4} \text { BER at } \\
15 \mathrm{~dB} \text { SNR for }\end{array}$ & Fading channel \\
\hline \multirow[t]{5}{*}{ Permutation } & [36] & $\begin{array}{l}\text { Codebook Design by QAM Constellation Segmentation: } \\
\text { Maximise MED by increasing the constellation layers, } \\
\text { creating an amplified effect in subsets, improving } \\
\text { decoding }\end{array}$ & $\begin{array}{l}\text { Achieves } 10^{-4} \text { BER at } \\
\text { 10dB SNR }\end{array}$ & $\begin{array}{l}\text { Fading channel } \\
\text { with LDPC } \\
\text { coding }\end{array}$ \\
\hline & [37] & $\begin{array}{l}\text { Codebook design using the phase rotation: Codebook } \\
\text { designed for user } 1 \text { using interleaving rotation. } \\
\text { Subsequent codebooks are created by rotating user } 1 \\
\text { codewords keeping MED constant }\end{array}$ & -- & $\begin{array}{l}\text { AWGN } \\
\text { Channel }\end{array}$ \\
\hline & [38] & $\begin{array}{l}\text { Codebook design using star-QAM constellations: Using } \\
\text { Star QAM Constellation with increasing the number of } \\
\text { layers, MED is improved. The method suggests an } \\
\text { increase as codebook size increases comparing with LDS }\end{array}$ & $\begin{array}{l}\text { Achieves } 10^{-4} \text { BER at } \\
\mathrm{E}_{\mathrm{b}} / \mathrm{N}_{\mathrm{o}}=16 \mathrm{~dB} \text { for } 8 \\
\text { array }(\mathrm{AWGN}), 24 \mathrm{~dB} \\
\text { for } 16 \text { array codebooks }\end{array}$ & $\begin{array}{l}\text { AWGN and } \\
\text { Fading channel }\end{array}$ \\
\hline & [39] & $\begin{array}{l}\text { Computer-generated-SCMA codebook: The method } \\
\text { maximises the MED for star QAM constellation by sub } \\
\text { partitioning the constellation using Trellis Coded } \\
\text { Modulation. }\end{array}$ & $\begin{array}{l}\text { Achieves } 10^{-4} \text { BER at } \\
8-10 \mathrm{~dB} \text { SNR for three } \\
\text { codebooks }\end{array}$ & $\begin{array}{l}\text { AWGN } \\
\text { Channel }\end{array}$ \\
\hline & [40] & $\begin{array}{l}\text { Codebook design base on circular-QAM: Reduce MPA } \\
\text { complexity by limiting projection points per signal tone, } \\
\text { placing codewords at coordinate points and origin. }\end{array}$ & $\begin{array}{l}\text { Achieves } 10^{-4} \text { BER at } \\
6 \mathrm{~dB} \text { SNR for four } \\
\text { array codebooks }\end{array}$ & $\begin{array}{l}\text { Fading channel } \\
\text { (convolutional } \\
\text { coding) }\end{array}$ \\
\hline \multirow[t]{3}{*}{$\begin{array}{l}\text { Factor Graph } \\
\text { Optimisation }\end{array}$} & [41] & $\begin{array}{l}\text { Dimension distance-based codebook: Achieves MED } \\
\text { improvement using turbo trellis, phase rotation, and IQ } \\
\text { interleaving. Select the best permutation set for diversity. }\end{array}$ & $\begin{array}{l}\text { Achieves } 10^{-4} \text { BER at } \\
11 \mathrm{~dB} \text { SNR for } 6 \text { and } \\
12 \mathrm{~dB} \text { SNR for } 8 \text { users }\end{array}$ & Fading channel \\
\hline & [42] & $\begin{array}{l}\text { Codebook Design for Large-Scale SCMA: Design } \\
\text { Largescale SCMA codebooks by using QAM with } \\
\text { random parameters }\end{array}$ & & \\
\hline & [43] & $\begin{array}{l}\text { Codebook Construction using Extended Factor Graph: } \\
\text { The design incorporates factor graphs of several RBs to } \\
\text { get the codebook. }\end{array}$ & & \\
\hline
\end{tabular}


b) Constellation rotation with permutation: The primary method in this category is given by Cai using rotation and interleaving for MD constellation optimisation [7]. The method rotates an initially produced lattice to generate multi-dimensions used for creating codebooks. It also uses interleaving for improving communication in fading channels. The study compares results with LDS; however, performance has minor improvements then LDS. A similar approach is followed by Bonilla [37], which incorporates the phase rotation and interleaving and a MED based detector in the receiver. Liu [36] suggested segmentation and combining based on the factor graph for MED improvement. Results show that Irregular LDS has quite comparative results at higher SNR as the proposed method, which signifies the importance of an irregular factor graph in LDS. Yu presented Star-QAM based codebook design [38] with a focus on improving MED. It includes designing four constellation point vectors that map to the elements of an nth dimension star constellation. Mapping vectors provide the mother codebook mathematically. The results have shown marked improvement from LDS but a minor improvement compared to the original SCMA. Yasmine also worked with star QAM constellations and proposed improvement in MED values using trellis coded modulation (TCM) [39]. Metkarunchit presented optimisation in receiver architecture by reducing MPA complexity [40]. The technique uses circular QAM where the number of projections is reduced to simplify MPA. The method, although less complicated, shows improvement with a small codebook. However, performance is reduced with 16 array codebooks due to MED.

c) Factor graph optimisation: Improvements based on information exchange and transfer efficiency is carried out in several methods. Yan work uses turbo trellis, phase rotation, and interleaving for MED and diversity improvement [41], improving BER performance and MPA performance by efficiently increasing the first MPA's convergence ability. Yang also designed largescale SCMA codebooks based on QAM with random parameters [42]. It generates a mapping matrix for every user, designing a codebook based on generated mapping matrix and layer operators using the mother constellation. Zhong presented factor graph-based block diversity improvement in design using an extended factor graph-based codebook [43]. This technique incorporates factor graphs of several RBs, interconnecting their nodes for diversity and performance.

\section{DISCUSSION}

Results show their effectiveness of codebook optimisation in different environments. Like codebook based on constellation rotation and interleaving based technique in [7] is presented to have a better BER then [24]. However, being a more straightforward technique, the required SNR for decoding is very high. Constellation segmentation in [36] is another technique with proper MED maximisation operation and better SNR gains. However, this technique's performance increases at higher SNRs, while matching results with other SCMA codebooks are observed at lower SNR values. The method in [31] is up-and-coming with low SNR signals but problematic in higher overloading situations due to erroneous multi-user detection. Compared with [41, 44, 45], it has a better BER performance and applicable to large codebooks than other techniques. The author in [37] signifies the importance of SNR with BER for phase rotation based codebook. Results show a drastic increase in errors with SNR higher than $-4 \mathrm{~dB}$. The results indicate the importance of further research in the phase rotated mother constellation design for practical purposes. The article [38] presents the proposed codebook's comparative results, with [24, 34, 45] showing better BER performance for large codebooks. [40] offers a circular QAM based codebook with an improved performance from the original SCMA codebook design [24]. The author in [35] presents a bit interleaved technique for codebook design with improved performance at much lower SNR. Methods in [32-34, 43] provide efficient codebook designing techniques with BER analysis in varying conditions and parameters.

This study analysed the codebooks from [33, 34, 36, 37, 39] and evaluated them for BER performance. The simulation is performed with six users and four resources, covering SNR signals from 4.7 to $22.7 \mathrm{~dB}$, including AWGN and fading channel with no channel coding. The code length is adapted to be 10,000 .

Figure 2 shows the simulation results of RBs with the best values for different codebooks. It is observed that codebooks from the rotation and permutation category have comparatively better performance. Considering the cumulative result of all RBs for a codebook, the codebook from [36] is observed to have a better error rate in both the lower and higher SNR. However, considering individual blocks, the star QAM codebook [39] is found to have the best results in some RBs. In both codebooks [36, 39], the proposed method is based on the prominent constellation segmentation where actual optimisation is performed. The constellation is then used for designing individual codebooks. It gives a direction of using segmentation in other methods presented, predicting for even better BER. 


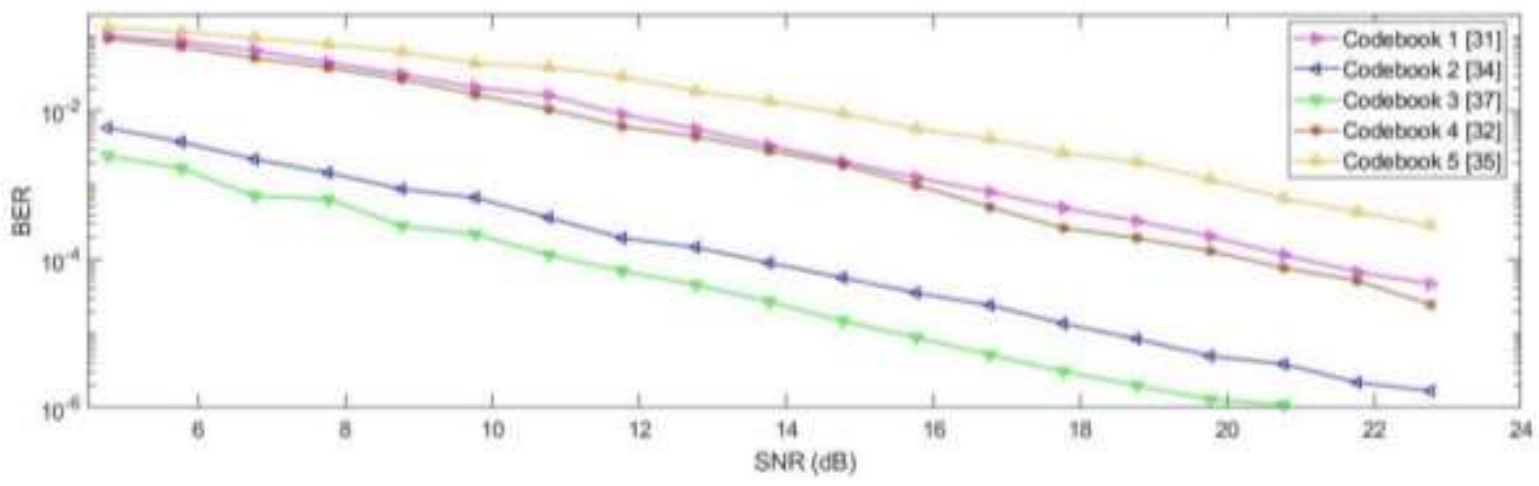

Figure 2. BER performance for SCMA system with different codebooks

\section{OPPORTUNITIES AND CHALLENGES}

The main challenge in codebook designing is user data segregation in signal space, optimising easy decoding and a simple decoder at the receiver [46]. In designing mother constellations, more power-efficient and simple models need to be developed, focusing on IoT [47]. There is a need to explore better-fit mathematical models to extract the required codebooks. Some theories are discussed in this study, but there is a need to explore mathematical models for codebook designing.

Further research should explore machine-learning techniques for SCMA data processing used in similar domains [48]. Prominent of these are regression, decision tree, and neural networks. Learning techniques can extract codebooks best suited for the required channel conditions. Machine learning can be enhanced by mixing with adaptive processing algorithms. Such a method exists in the 5G domain as presented in [48], and they can be used for the SCMA domain. According to channel or data, adaptive nature can be explored, leading to a new codebook domain for $5 \mathrm{G}$ communication. The author utilised an adaptive approach in designing better SCMA architectures according to user features [49]. Another research domain is resource planning organisation [50], including adaptively allocating resources and developing optimised algorithms.

\section{CONCLUSION}

The techniques presented in designing and optimising the codebook aim to provide better BER and efficient user detection. The study reviews various methods existing in the literature for codebook designing. Methods are categorised based on their approach, and performance is evaluated considering multiple parameters. Future work should focus more on the MD constellations adding complex mathematical models or machine learning, including neural networks, to have even better constellations.

\section{ACKNOWLEDGEMENTS}

This study was funded through UTM Research Grant-R. K130000.2656.18J05

\section{REFERENCES}

[1] M. Nirmala, K. Thirupathi Raja, and S. Sukirtha, "Review of transformation of mobile wireless for next generation communication networks," International Journal of Innovative Technology and Exploring Engineering, vol. 8, pp. 292-295, 01/01 2018.

[2] L. J. Vora, "Evolution of mobile generation technology: 1G to 5G and review of upcoming wireless technology 5G," International journal of modern trends in engineering and research, vol. 2, no. 10, pp. 281-290, 2015.

[3] J. Goyal, K. Singla, and S. Singh, "A Survey of Wireless Communication Technologies from 1G to 5G," in International Conference on Computer Networks and Inventive Communication Technologies, 2019, pp. 613-624: Springer, doi: 10.1007/978-3-030-37051-0_69

[4] S. Shukla, V. Khare, S. Garg, and P. Sharma, "Comparative Study of 1G, 2G, 3G and 4G," J. Eng. Comput. Appl. Sci, vol. 2, no. 4, pp. 55-63, 2013.

[5] M. L. Roberts, M. A. Temple, R. F. Mills, and R. A. Raines, "Evolution of the air interface of cellular communications systems toward 4G realisation," IEEE Communications Surveys \& Tutorials, vol. 8, no. 1, pp. 223, 2006, doi: 10.1109/COMST.2006.323439

[6] H. Yin and S. Alamouti, "OFDMA: A broadband wireless access technology," in 2006 IEEE sarnoff symposium, 2006, pp. 1-4: IEEE, doi: 10.1109/SARNOF.2006.4534773 
[7] D. Cai, P. Fan, X. Lei, Y. Liu, and D. Chen, "Multi-dimensional SCMA codebook design based on constellation rotation and interleaving," in 2016 IEEE 83rd Vehicular Technology Conference (VTC Spring), 2016, pp. 1-5: IEEE, doi: 10.1109/VTCSpring.2016.7504356

[8] Y. Liu, Z. Qin, M. Elkashlan, Z. Ding, A. Nallanathan, and L. Hanzo, "Non-orthogonal multiple access for 5G and beyond," Proceedings of the IEEE, vol. 105, no. 12, pp. 2347-2381, 2017, doi: 10.1109/JPROC.2017.2768666

[9] L. Dai, B. Wang, Y. Yuan, S. Han, I. Chih-Lin, and Z. Wang, "Non-orthogonal multiple access for 5G: solutions, challenges, opportunities, and future research trends," IEEE Communications Magazine, vol. 53, no. 9, pp. 74-81, 2015, doi: 10.1109/MCOM.2015.7263349

[10] L. Dai, B. Wang, Z. Ding, Z. Wang, S. Chen, and L. Hanzo, "A survey of non-orthogonal multiple access for 5G," IEEE communications surveys \& tutorials, vol. 20, no. 3, pp. 2294-2323, 2018, doi: 10.1109/COMST.2018.2835558

[11] L. P. Liyn et al., "Ant-colony and nature-inspired heuristic models for NOMA systems: a review," TELKOMNIKA, vol. 18, no. 4, pp. 1754-1761, 2020, doi: 10.12928/telkomnika.v18i4.14995

[12] S. R. Islam, N. Avazov, O. A. Dobre, and K.-S. Kwak, "Power-domain non-orthogonal multiple access (NOMA) in 5G systems: Potentials and challenges," IEEE Communications Surveys \& Tutorials, vol. 19, no. 2, pp. 721-742, 2016, doi: 10.1109/COMST.2016.2621116

[13] Y. Cai, Z. Qin, F. Cui, G. Y. Li, and J. A. McCann, "Modulation and multiple access for 5G networks," IEEE Communications Surveys \& Tutorials, vol. 20, no. 1, pp. 629-646, 2017, doi: 10.1109/COMST.2017.2766698

[14] M. Moltafet, N. M. Yamchi, M. R. Javan, and P. Azmi, "Comparison study between PD-NOMA and SCMA," IEEE Transactions on Vehicular Technology, vol. 67, no. 2, pp. 1830-1834, 2017, doi: 10.1109/TVT.2017.2759910

[15] M. Qiu, Y.-C. Huang, S.-L. Shieh, and J. Yuan, "A lattice-partition framework of downlink non-orthogonal multiple access without SIC," IEEE Transactions on Communications, vol. 66, no. 6, pp. 2532-2546, 2018, doi: 10.1109/TCOMM.2018.2805895

[16] Y. Tao, L. Liu, S. Liu, and Z. Zhang, "A survey: Several technologies of non-orthogonal transmission for 5G," China communications, vol. 12, no. 10, pp. 1-15, 2015, doi: 10.1109/CC.2015.7315054

[17] Z. Wu, K. Lu, C. Jiang, and X. Shao, "Comprehensive study and comparison on 5G NOMA schemes," IEEE Access, vol. 6, pp. 18511-18519, 2018, doi: 10.1109/ACCESS.2018.2817221

[18] I. Parvez, A. Rahmati, I. Guvenc, A. I. Sarwat, and H. Dai, "A survey on low latency towards 5G: RAN, core network and caching solutions," IEEE Communications Surveys \& Tutorials, vol. 20, no. 4, pp. 3098-3130, 2018, doi: 10.1109/COMST.2018.2841349

[19] M. B. Shahab, R. Abbas, M. Shirvanimoghaddam, and S. J. Johnson, "Grant-free Non-orthogonal Multiple Access for IoT: A Survey," arXiv preprint arXiv:1910.06529, 2019, doi: 10.1109/COMST.2020.2996032

[20] M. Vameghestahbanati, I. D. Marsland, R. H. Gohary, and H. Yanikomeroglu, "Multidimensional constellations for uplink SCMA systems-A comparative study," IEEE Communications Surveys \& Tutorials, vol. 21, no. 3, pp. 21692194, 2019, doi: 10.1109/COMST.2019.2910569

[21] H. Nikopour and H. Baligh, "Sparse code multiple access," in 2013 IEEE 24th Annual International Symposium on Personal, Indoor, and Mobile Radio Communications (PIMRC), 2013, pp. 332-336: IEEE, doi: 10.1109/PIMRC.2013.666615

[22] B. Xiao, K. Xiao, S. Zhang, Z. Chen, B. Xia, and H. Liu, "Iterative detection and decoding for SCMA systems with LDPC codes," in 2015 International Conference on Wireless Communications \& Signal Processing (WCSP), 2015, pp. 1-5: IEEE, doi: 10.1109/WCSP.2015.7341325

[23] M. Vameghestahbanati, I. Marsland, R. H. Gohary, and H. Yanikomeroglu, "Polar codes for SCMA systems," in 2017 IEEE 86th Vehicular Technology Conference (VTC-Fall), 2017, pp. 1-5: IEEE.

[24] M. Taherzadeh, H. Nikopour, A. Bayesteh, and H. Baligh, "SCMA codebook design," in 2014 IEEE 80th Vehicular Technology Conference (VTC2014-Fall), 2014, pp. 1-5: IEEE, doi: 10.1109/VTCFall.2014.6966170

[25] E. Biglieri, J. Proakis, and S. Shamai, "Fading channels: Information-theoretic and communications aspects," IEEE transactions on information theory, vol. 44, no. 6, pp. 2619-2692, 1998, doi: 10.1109/18.720551

[26] L. Tian, J. Zhong, M. Zhao, and L. Wen, "A suboptimal algorithm for SCMA codebook design over uplink Rayleigh fading channels," in 2018 IEEE 87th Vehicular Technology Conference (VTC Spring), 2018, pp. 1-5: IEEE, doi: 10.1109/VTCSpring.2018.8417528

[27] P. Robertson, E. Villebrun, and P. Hoeher, "A comparison of optimal and sub-optimal MAP decoding algorithms operating in the log domain," in Proceedings IEEE International Conference on Communications ICC'95, 1995, vol. 2, pp. 1009-1013: IEEE, doi: 10.1109/ICC.1995.524253

[28] K. Xiao, B. Xiao, S. Zhang, Z. Chen, and B. Xia, "Simplified multiuser detection for SCMA with sum-product algorithm," in 2015 International Conference on Wireless Communications \& Signal Processing (WCSP), 2015, pp. 1-5: IEEE, doi: 10.1109/WCSP.2015.7341328

[29] J. Dommel, Z. Utkovski, L. Thiele, and S. Stańczak, "Sparse Code-Domain Non-Orthogonal Random Access with Peeling Decoder," in 2019 53rd Asilomar Conference on Signals, Systems, and Computers, 2019, pp. 984-988: IEEE, doi: 10.1109/IEEECONF44664.2019.9049075

[30] X. Zhang, D. Zhang, L. Yang, G. Han, H.-H. Chen, and D. Zhang, "Uniquely Decomposable Constellation Group for SCMA Codebook Design," arXiv preprint arXiv:2103.00126, 2021.

[31] X. Zhang, G. Han, D. Zhang, and L. Yang, "A Lattice-Based SCMA Codebook Design for IoMT Communications," in 2019 IEEE/CIC International Conference on Communications Workshops in China (ICCC Workshops), 2019, pp. 169-173: IEEE, doi: 10.1109/ICCChinaW.2019.8849945 
[32] C. Dong, G. Gao, K. Niu, and J. Lin, "An Efficient SCMA Codebook Optimization Algorithm Based on Mutual Information Maximization," Wireless Communications and Mobile Computing, vol. 2018, 2018, doi: $10.1155 / 2018 / 8910907$

[33] Y. Zhang et al., "Constant Modulus Codebook Design for SCMA System," in 2018 IEEE International Conference on Communication Systems (ICCS), 2018, pp. 242-246: IEEE, doi: 10.1109/ICCS.2018.8689235

[34] S. Zhang et al., "A capacity-based codebook design method for sparse code multiple access systems," in 2016 8th International Conference on Wireless Communications \& Signal Processing (WCSP), 2016, pp. 1-5: IEEE, doi: 10.1109/WCSP.2016.7752620

[35] J. Bao, Z. Ma, M. Xiao, T. A. Tsiftsis, and Z. Zhu, "Bit-interleaved coded SCMA with iterative multiuser detection: Multidimensional constellations design," IEEE Transactions on Communications, vol. 66, no. 11, pp. 5292-5304, 2017, doi: 10.1109/TCOMM.2017.2782325

[36] S. Liu, J. Wang, J. Bao, and C. Liu, "Optimised SCMA Codebook Design by QAM Constellation Segmentation With Maximized MED," IEEE Access, vol. 6, pp. 63232-63242, 2018, doi: 10.1109/ACCESS.2018.2876030

[37] J. L. L. Bonilla, S. V. Beltrán, I. S. Rivera, and F. M. Piñón, "Construction of SCMA CodeBooks using the phase rotation method," in 2018 IEEE International Autumn Meeting on Power, Electronics and Computing (ROPEC), 2018, pp. 1-8: IEEE, doi: 10.1109/ROPEC.2018.8661414

[38] L. Yu, P. Fan, D. Cai, and Z. Ma, "Design and analysis of SCMA codebook based on star-QAM signaling constellations," IEEE Transactions on Vehicular Technology, vol. 67, no. 11, pp. 10543-10553, 2018, doi: 10.1109/TVT.2018.2865920

[39] Y. M. Tabra and B. M. Sabbar, "New Computer Generated-Scma Codebook With Maximised EuclidiaN Distance For 5G," Iraqi Journal of Information \& Communications Technology, vol. 2, no. 2, pp. 9-24, 2019, doi: 10.31987/ijict.2.2.64

[40] T. Metkarunchit, "SCMA codebook design base on circular-QAM," in 2017 Integrated Communications, Navigation and Surveillance Conference (ICNS), 2017, pp. 3E1-1-3E1-8: IEEE, doi: 10.1109/ICNSURV.2017.8011917

[41] C. Yan, G. Kang, and N. Zhang, "A dimension distance-based SCMA codebook design," IEEE Access, vol. 5, pp. 5471-5479, 2017, doi: 10.1109/ACCESS.2017.2685618

[42] C. Yang, S. Jing, X. Liang, Z. Zhang, X. You, and C. Zhang, "A Low-Complexity Codebook Design for LargeScale SCMA," in 2018 IEEE Asia Pacific Conference on Circuits and Systems (APCCAS), 2018, pp. 195-198: IEEE, doi: 10.1109/APCCAS.2018.8605704

[43] C. Zhong, K. Niu, J. Dai, and C. Dong, "A Novel SCMA Codebook Construction Based on Extended Factor Graph Design," in 2018 IEEE Globecom Workshops (GC Wkshps), 2018, pp. 1-6: IEEE, doi: 10.1109/GLOCOMW.2018.8644434

[44] Y. Zhou, Q. Yu, W. Meng, and C. Li, "SCMA codebook design based on constellation rotation," in 2017 IEEE International Conference on Communications (ICC), 2017, pp. 1-6: IEEE, doi: 10.1109/ICC.2017.7996395

[45] L. Yu, X. Lei, P. Fan, and D. Chen, "An optimised design of SCMA codebook based on star-QAM signaling constellations," in 2015 International Conference on Wireless Communications \& Signal Processing (WCSP), 2015, pp. 1-5: IEEE, doi: 10.1109/WCSP.2015.7341311

[46] F. Wei and W. Chen, "Low complexity iterative receiver design for sparse code multiple access," IEEE Transactions on Communications, vol. 65, no. 2, pp. 621-634, 2016, doi: 10.1109/TCOMM.2016.2631468

[47] A. Alnoman, S. Erkucuk, and A. Anpalagan, "Sparse code multiple access-based edge computing for IoT systems," IEEE Internet of Things Journal, vol. 6, no. 4, pp. 7152-7161, 2019, doi: 10.1109/JIOT.2019.2914570

[48] D. A. Awan, R. L. Cavalcante, M. Yukawa, and S. Stanczak, "Detection for 5G-NOMA: An online adaptive machine learning approach," in 2018 IEEE International Conference on Communications (ICC), 2018, pp. 1-6: IEEE, doi: 10.1109/ICC.2018.8422449

[49] D. Zhai, "Adaptive codebook design and assignment for energy saving in SCMA networks," IEEE Access, vol. 5, pp. 23550-23562, 2017, doi: 10.1109/ACCESS.2017.2764120

[50] S. Han, C. Guo, W. Meng, and C. Li, "A flexible resource scheduling scheme for an adaptive SCMA system," Computer Networks, vol. 129, pp. 384-391, 2017. 